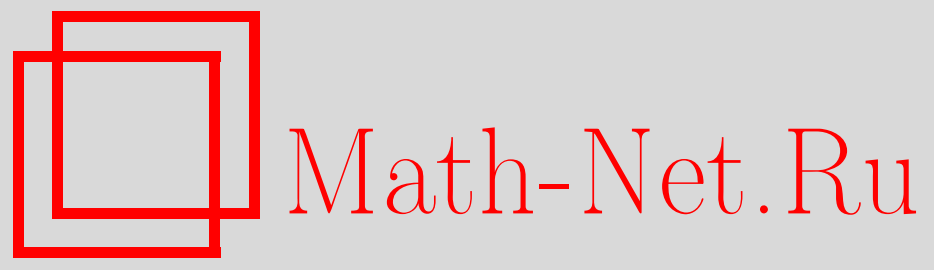

E. I. Pancheva, E. T. Kolkovska, P. K. Jordanova, Random time-changed extremal processes, Теория вероятн. и ее примен., 2006, том 51, выпуск 4, 752-772 DOI: https://doi.org/10.4213/tvp23

Использование Общероссийского математического портала Math-Net.Ru подразумевает, что вы прочитали и согласны с пользовательским соглашением

http: //www . mathnet.ru/rus/agreement

Параметры загрузки:

IP: 52.6 .47 .48

26 апреля 2023 г., 15:33:52

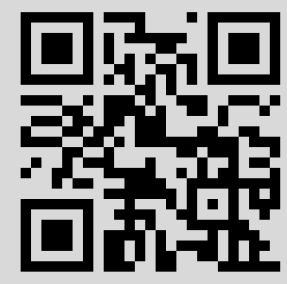




\section{RANDOM TIME-CHANGED EXTREMAL PROCESSES}

Точечный процесс $\mathscr{N}=\left\{\left(T_{k}, X_{k}\right): k \geqslant 1\right\}$, который мы рассматриваем, предполагается бернуллиевским с независимыми случайными векторами $X_{k}$ в $[0, \infty)^{d}$ и случайными моментами времени $T_{k}$ в $[0, \infty)$, независимыми от $X$. Для нормировки мы используем регулярную последовательность $\xi_{n}(t, x)=\left(\tau_{n}(t), u_{n}(x)\right)$ замены времени-пространства со значениями в $[0, \infty)^{1+d}$. Мы рассматриваем последовательность ассоциированных экстремальных процессов

$$
\tilde{Y}_{n}(t)=\left\{\bigvee u_{n}^{-1}\left(X_{k}\right): T_{k} \leqslant \tau_{n}(t)\right\}
$$

где операция взятия максимума « $\vee \gg$ определена в $\mathbf{R}^{d}$ покомпонентно. Мы предполагаем, что существуют стохастически непрерывный временной процесс $\theta=\{\theta(t): t \geqslant 0\}$, строго возрастающий и независимый от $\left\{X_{k}\right\}$, и целочисленная детерминированная считающая функция $k$ на $[0, \infty)$ такие, что считающий процесс $N$ точечного процесса $\mathscr{N}$ имеет вид $N(s)=k(\theta(s))$ п.н.

В такой постановке мы доказываем функциональную теорему переноса, которая утверждает, что если $\tau_{n}^{-1} \circ \theta \circ \tau_{n} \Rightarrow \Lambda$, где $\Lambda$ строго возрастает и является стохастически непрерывным, и $\bigvee_{k=1}^{k\left(\tau_{n}(\cdot)\right)} u_{n}^{-1}\left(X_{k}\right) \Rightarrow Y(\cdot)$, то $\tilde{Y}_{n} \Rightarrow \tilde{Y}=Y \circ \Lambda$, где $Y-$ caмоподобный экстремальный процесс. Мы называем такие предельные процессы процессами со случайной заменой времени или составными процессами. Они являются стохастически непрерывными и самоподобными относительно той же самой однопараметрической нормируюшей группы, что и $Y$. Мы показываем, что составной процесс является экстремальным (т.е. процессом с независимыми max-прирашениями) тогда и только тогда, когда $\Lambda$ имеет независимые приращения и $Y$ имеет однородные max-приращения. В заключение мы применяем случайные экстремальные процессы с заменой времени для нахождения оценки снизу вероятности разорения в связанной с $\mathscr{N}$ страховой модели. Мы даем также оценку сверху, получаемую с помошью $\alpha$-устойчивого движения Леви.

Ключевые слова и фразы: экстремальные процессы, слабые предельные теоремы, вероятность разорения.

* IMI-BAS, Sofia, Bulgaria; e-mail: pancheva@math.bas.bg

** CIMAT, Guanajuato, Mexico.

*** University of Shumen, Shumen, Bulgaria. 
1. Introduction. In this section we recall some definitions and basic facts used in the paper.

Time-space Bernoulli point processes $\mathscr{N}=\left\{\left(T_{k}, X_{k}\right): k \geqslant 1\right\}$ were introduced in $[2$, section 7$]$. They are point processes defined on a locally compact metric space $\mathscr{S}$ and satisfying the following conditions:

i) their mean measure $\mu$ is a Radon measure on $\mathscr{S}$ (i.e., finite on compact subsets of $\mathscr{S}$ );

ii) they are simple in time: $T_{k} \neq T_{j}$ a.s. for $k \neq j$;

iii) restrictions of $\mathscr{N}$ to slices over disjoint time intervals are independent.

Such point processes are important in Extreme Value Theory: In fact, any extremal process $Y:[0, \infty) \rightarrow[0, \infty)^{d}$ is generated by an increasing rightcontinuous curve $C$, the lower curve of $Y$ (see [2] for details), and a Bernoulli point process $\mathscr{N}$ on $\mathscr{S}=[0, C]^{c}$ :

$$
Y(t)=C(t) \vee\left\{\vee X_{k}: T_{k} \leqslant t\right\} .
$$

Here $\left\{T_{k}\right\}$ are distinct random time points and $\left\{X_{k}\right\}$ are independent random vectors in $[0, \infty)^{d}$. The operation maximum $\vee$ as well as equalities and inequalities in $\mathbf{R}^{d}$ are understood componentwise, and $A^{c}$ denotes the complement of the set $A$ in $[0, \infty)^{d+1}$.

An extremal process $Y$ is infinitely divisible with respect to the operation maximum, briefly max-id, if for every $n \geqslant 1$ there exist independent and identically distributed (i.i.d.) extremal processes $Y_{1 n}, \ldots, Y_{n n}$ such that $Y \stackrel{\text { fdd }}{=} Y_{1 n} \vee \cdots \vee Y_{n n}$. The max-infinite divisibility of random vectors in $\mathbf{R}^{d}$ is investigated first in [1]. In [2] the authors prove that an extremal process $Y:[0, \infty) \rightarrow[0, \infty)^{d}$ is max-id if and only if the associated Bernoulli point process is Poisson. In this case there is a simple connection between the distribution function $f$ of $Y$, where $f(t, x)=\mathbf{P}\{Y(t)<x\}$, and the mean measure $\mu$ of $\mathscr{N}$, where $\mu(A)=\mathbf{E} \mathscr{N}(A), A \subset \mathscr{S}$, namely

$$
f(t, x)=\exp \left\{-\mu\left([0, t] \times A_{x}^{c}\right)\right\}, \quad x>C(t), \quad t>0,
$$

with $A_{x}=\left\{y \in[0, \infty)^{d}: y<x\right\}$. Note that $\mu(A)<\infty$ for all $A$ of the form $[0, t] \times A_{x}^{c}$ as long as $x>C(t)$, whereas $[0, C]$ is the explosion area of $\mu$.

Let $\mathscr{M}^{*}([0, \infty))$ be the space of all right-continuous increasing functions $y:[0, \infty) \rightarrow[0, \infty)^{d}, y(t)<\infty, y(t) \rightarrow \vec{\infty}$ for $t \rightarrow \infty, \vec{\infty}=(\infty, \ldots, \infty)$. So, the sample paths of any extremal process belong to $\mathscr{M}^{*}$ a.s. Given a sequence of extremal processes $\left\{Y_{n}\right\}, Y_{n}:[0, \infty) \rightarrow[0, \infty)^{d}$, we denote the distribution function of $Y_{n}$ and the probability distribution of $Y_{n}$ on $\mathscr{M}^{*}$ by $f_{n}$ and $\pi_{n}$, respectively. For fixed $t>0$, let $F_{n, t}(\cdot):=f_{n}(t, \cdot)$. We say the sequence $\left\{Y_{n}\right\}$ is weakly convergent to an extremal process $Y:[0, \infty) \rightarrow$ $[0, \infty)^{d}$ with distribution function $f$ and probability distribution $\pi$, and write $Y_{n} \Rightarrow Y$, if one of the following equivalent statements is met [2, Theorem 6.1]:

1) $f_{n} \rightarrow f$ at all continuity points of $f$; 
2) $F_{n, t} \rightarrow F_{t}:=f(t, \cdot)$ weakly for each $t$ in a dense subset of $(0, \infty)$;

3) $\int \phi d \pi_{n} \rightarrow \int \phi d \pi$ for bounded $\phi: \mathscr{M}^{*}([0, \infty)) \rightarrow \mathbf{R}$ which are continuous in the weak topology of $\mathscr{M}^{*}$.

Recall that the univariate marginals of an extremal process determine its finite dimensional distributions. If additionally to $Y_{n} \stackrel{\text { fdd }}{\longrightarrow} Y$ we assume that the limit extremal process $Y$ is stochastically continuous, then $Y_{n} \Rightarrow Y$ also in the Skorokhod topology of $\mathscr{M}^{*}$ (see, e.g., [3, Theorem 3]).

A mapping $\xi(t, x)=(\tau(t), u(x)), t \in[0, \infty), x \in[0, \infty)^{d}$, strictly increasing and continuous in each coordinate is called time-space change of $[0, \infty)^{1+d}$. An increasing in $n$ sequence of time-space changes $\left\{\xi_{n}\right\}$ is referred to as regular if for any $s>0$ there exists a time-space change $\eta_{s}(t, x)=\left(\sigma_{s}(t), U_{s}(x)\right)$ so that

$$
\tau_{n}^{-1} \circ \tau_{[n s]}(t) \rightarrow \sigma_{s}(t), \quad u_{n}^{-1} \circ u_{[n s]}(x) \rightarrow U_{s}(x)
$$

pointwise and the correspondence $s \leftrightarrow \eta_{s}$ is one-to-one. Then the family $\mathscr{L}=\left\{\eta_{s}: s>0\right\}$ forms a continuous one-parameter group with respect to composition (cf. [10] and also [11] for details).

Consider the following model (A): let $X:[0, \infty) \rightarrow[0, \infty)^{d}$ be an extremal process with lower curve $C$ and associated Bernoulli point process $\mathscr{N}=\left\{\left(t_{k}, X_{k}\right): k \geqslant 1\right\}, t_{k}$ distinct and nonrandom, $X_{k}$ independent with distribution function which does not have defect at $+\infty$. We assume that there is a regular norming sequence $\xi_{n}(t, x)=\left(\tau_{n}(t), u_{n}(x)\right)$ of time-space changes of $[0, \infty)^{1+d}$ so that the sequence of extremal processes

$$
Y_{n}(t):=u_{n}^{-1} \circ X \circ \tau_{n}(t)=C_{n}(t) \vee\left\{\bigvee u_{n}^{-1}\left(X_{k}\right): t_{k} \leqslant \tau_{n}(t)\right\}
$$

is weakly convergent to a nondegenerate extremal process $Y$ with distribution function $f$ and $Y(0)=0$ a.s. Then the limit extremal process is stochastically continuous for all $t \geqslant 0$ and self-similar with respect to $\mathscr{L}$, i.e., $Y$ satisfies

$$
U_{s} \circ Y(t) \stackrel{\mathrm{d}}{=} Y \circ \sigma_{s}(t) \quad \forall s>0
$$

or equivalently

$$
f(t, x)=f\left(\sigma_{s}(t), U_{s}(x)\right) \quad \forall s>0 .
$$

The paper [10] is devoted to studying the properties of self-similar extremal processes. One of them is the fact that the univariate marginals of $Y$ are max-selfdecomposable. If additionally the initial extremal process $X$ has homogeneous max-increments, then the limit process $Y$ is max-stable. Another important property of a self-similar extremal process is that its lower curve is continuous.

As known, the self-similar extremal processes form a special subclass of the semi-selfsimilar extremal processes studied in [11]. The latter processes satisfy characteristic equation (1.2) for only one fixed $s_{0} \in(0, \infty)$ rather than for all $s>0$. 
In the present paper we change the previous model (A) by the model (B), where we assume that the time points $T_{k}$ of a given Bernoulli point process $\widetilde{\mathcal{N}}=\left\{\left(T_{k}, X_{k}\right): k \geqslant 1\right\}$ are random variables in $[0, \infty)$ and the space points $X_{k}$ are i.i.d. random vectors in $[0, \infty)^{d}$. Note, since $X_{k}$ are i.i.d., the lower curve $C(t)$ of the associated with $\widetilde{\mathscr{N}}$ extremal process

$$
X(t)=C(t) \vee\left\{\bigvee X_{k}: T_{k} \leqslant t\right\}
$$

is constant. Hence we may and do assume $C(t) \equiv 0$. Consequently, for all $n \geqslant 1$ the lower curve $C_{n}$ of $\tilde{Y}_{n}(t)=u_{n}^{-1} \circ X \circ \tau_{n}(t)$ is zero too. In section 2 we are concerned with the following questions.

1. Under which conditions on the Bernoulli point process $\widetilde{\mathscr{N}}$ the sequence of the associated extremal processes

$$
\tilde{Y}_{n}(t)=\left\{\bigvee u_{n}^{-1}\left(X_{k}\right): T_{k} \leqslant \tau_{n}(t)\right\}
$$

is weakly convergent to a nondegenerate process $\tilde{Y}$ ?

2. Which class does $\tilde{Y}$ belong to?

We assume further that there exists a stochastically continuous time process $\theta(t), t \geqslant 0$, independent of $\left\{X_{k}\right\}$, such that the counting process $N$ of $\widetilde{N}, N(t)=\sum_{k} I_{[0, t]}\left(T_{k}\right)$, is of the form $k(\theta(t))$. Here $I_{A}$ is the indicator of the set $A$ and $k(t)$ is a deterministic counting function. In this framework our main result in section 2 is the Functional Transfer Theorem. It claims in general that if $Y_{n} \Rightarrow Y$ and $\tau_{n}^{-1} \circ \theta \circ \tau_{n} \Rightarrow \Lambda$ in $\mathscr{M}([0, \infty))$, then the limit process $\widetilde{Y}$ is of the form $\widetilde{Y}=Y \circ \Lambda$. Here $Y$ is a self-similar extremal process and $\Lambda:[0, \infty) \rightarrow[0, \infty)$ is a stochastically continuous time process independent of $Y$ and with a.s. strictly. increasing sample paths. We call such processes $\tilde{Y}$ random time-changed or compound self-similar extremal processes. The main contribution of our paper are their properties studied in section 3. In section 4 we use the compound extremal process to find a lower bound for the ruin probability in a particular insurance model. Furthermore, using a similar technics as in [4], we find an upper bound too.

Another aspects of random time-changed extremal processes can be found in [13].

2. Compound extremal process as limiting. Let us denote by $\mathscr{M}([0, \infty))$ the set of all strictly increasing right-continuous functions $\tau:[0, \infty) \rightarrow[0, \infty), \tau(0)=0, \tau(u) \rightarrow \infty$ as $u \rightarrow \infty$.

In this section we consider the following model (B): The point process $\widetilde{N}=\left\{\left(T_{k}, X_{k}\right): k \geqslant 1\right\}$ we deal with is assumed to be Bernoulli with i.i.d. random variables $X_{k}$ in $[0, \infty)^{d}$ and with random time points $T_{k}$ in $[0, \infty)$, independent of $X$. We suppose the latter to be ordered, $T_{1}<T_{2}<\cdots$, $T_{k} \rightarrow \infty$ a.s., and defined on the same probability space $[\Omega, \mathscr{A}, \mathbf{P}]$ as $X$. Denote by $N(t)$ the counting function of $\widetilde{N}$. We assume further that there exist a stochastically continuous time process $\theta:[0, \infty) \rightarrow[0, \infty)$ with sample 
paths in $\mathscr{M}([0, \infty))$ and a deterministic counting function $k$ on $[0, \infty)$ such that for $s>0$ and almost all (a.a.) $\omega \in \Omega$ it holds

$$
N(\omega, s)=k(\theta(\omega, s)) .
$$

Let $\left\{t_{k}: k \geqslant 1\right\}, t_{1}<t_{2}<\cdots$, be the nonrandom time point process whose counting function $k(t)=\sum_{k} I_{[0, t]}\left(t_{k}\right)$ coincides with the function $k(\cdot)$ of (2.1). Then

$$
\begin{gathered}
N(t)=\sum_{k} I_{[0, t]}\left(T_{k}\right) \stackrel{\text { a.s. }}{=} \sum_{k} I_{[0, \theta(t)]}\left(t_{k}\right)=k(\theta(t)), \\
\mathbf{P}\{N(t) \geqslant k\}=\mathbf{P}\left\{T_{k} \leqslant t\right\}=\mathbf{P}\left\{t_{k} \leqslant \theta(t)\right\} .
\end{gathered}
$$

$\mathrm{E} \times \mathrm{a} \mathrm{m} \mathrm{p} \mathrm{le} \mathrm{1.} \mathrm{Assume} \mathrm{that}\left\{T_{k}\right\}$ is a simple Poisson point process on $[0, \infty)$ with mean measure $\mathbf{E} N(t)=\lambda t, \lambda>0$. One can interpret $T_{k}$ as the arrival time of the $k$ th claim $X_{k}$ in a certain insurance model. Assume further that there is a deterministic counting process $k(t)$ such that the accumulated claim process $S(t)=\sum_{k=1}^{k(t)} X_{k}$, properly normalized, has a nondegenerate weak limit. Let $\left\{t_{0}=0, t_{1}, t_{2}, \ldots\right\}, t_{k} \rightarrow \infty$, be the point process associated with $k(t)$. We show that there exists a stochastically continuous time process $\theta(t)$ with sample paths in $\mathscr{M}$ such that $N(t)=k(\theta(t))$. Let us denote $Q_{t}(s)=\mathbf{P}\{\theta(t)<s\}$.

Indeed, since for every $t>0$

$$
\begin{aligned}
\mathbf{P}\{k(\theta(t))=n\} & =\sum_{k=0}^{\infty} \mathbf{P}\left\{k(\theta(t))=n, t_{k} \leqslant \theta(t)<t_{k+1}\right\} \\
& =\mathbf{P}\left\{t_{n} \leqslant \theta(t)<t_{n+1}\right\}=Q_{t}\left(t_{n+1}\right)-Q_{t}\left(t_{n}\right),
\end{aligned}
$$

we obtain the values of $Q_{t}(s)$ for $s \in\left\{t_{0}, t_{1}, t_{2}, \ldots\right\}$ by the iteration formula

$$
Q_{t}\left(t_{n+1}\right)-Q_{t}\left(t_{n}\right)=\frac{(\lambda t)^{n}}{n !} e^{-\lambda t}
$$

For $s \notin\left\{t_{0}, t_{1}, t_{2}, \ldots\right\}$ one can interpolate $Q_{t}(s)$ by preserving the properties required above.

Let us use a regular sequence $\xi_{n}(t, x)=\left(\tau_{n}(t), u_{n}(x)\right)$ of time-space changes of $[0, \infty)^{1+d}$ for normalizing, so that the sequence of the associated with $\widetilde{N}$ extremal processes

$$
\tilde{Y}_{n}(t)=\left\{\bigvee u_{n}^{-1}\left(X_{k}\right): T_{k} \leqslant \tau_{n}(t)\right\}
$$

is weakly convergent to a nondegenerate increasing process $\tilde{Y}$. We ask here which class does $\widetilde{Y}$ belong to?

Consider the point process $\mathscr{N}=\left\{\left(t_{k}, X_{k}\right): k \geqslant 1\right\}$ associated with $\widetilde{N}$ by (2.1). Using the same norming sequence as in (2.3), we form the sequence of point processes

$$
\mathscr{N}_{n}=\left\{\left(\tau_{n}^{-1}\left(t_{k}\right), u_{n}^{-1}\left(X_{k}\right)\right): k \geqslant 1\right\}
$$


with counting function

$$
k_{n}(t)=\sum_{k} I_{[0, t]}\left(\tau_{n}^{-1}\left(t_{k}\right)\right),
$$

and

$$
\widetilde{\mathscr{N}}_{n}=\left\{\left(\tau_{n}^{-1}\left(T_{k}\right), u_{n}^{-1}\left(X_{k}\right)\right): k \geqslant 1\right\}
$$

with a random counting function

$$
N_{n}(t)=\sum_{k} I_{[0, t]}\left(\tau_{n}^{-1}\left(T_{k}\right)\right)
$$

Since $\mathbf{P}\left\{\tau_{n}^{-1}\left(T_{k}\right) \leqslant t\right\}=\mathbf{P}\left\{\tau_{n}^{-1}\left(t_{k}\right) \leqslant \tau_{n}^{-1} \circ \theta \circ \tau_{n}(t)\right\}$ we see that $N_{n}(t) \stackrel{\mathrm{d}}{=}$ $k_{n}\left(\theta_{n}(t)\right)$ with $\theta_{n}(t)=\tau_{n}^{-1} \circ \theta \circ \tau_{n}(t)$.

The extremal process

$$
Y_{n}(t)=\left\{\bigvee_{k} u_{n}^{-1}\left(X_{k}\right): \tau_{n}^{-1}\left(t_{k}\right) \leqslant t\right\}
$$

associated with $\mathscr{N}_{n}$ and the extremal process $\widetilde{Y}_{n}$ associated with $\widetilde{\mathscr{N}}_{n}$, are connected by the relation $\widetilde{Y}_{n}(t) \stackrel{\mathrm{d}}{=} Y_{n}\left(\theta_{n}(t)\right)$. In this way we have reduced the convergence problem of $\widetilde{Y}_{n}$ to both the convergence of $Y_{n}$, considered in the previous section, and the convergence of $\theta_{n}$. To solve it we use the continuity of the composition in the weak topology in $D([0, \infty)$ ). (See Theorem 3 in [14] and the comments following the theorem; also consult [15].)

Proposition 2.1. Let $\left\{Y_{n}, n \geqslant 1\right\}$ be a sequence of extremal processes weakly convergent to a stochastically continuous extremal process $Y$. Let $\left\{\theta_{n}, n \geqslant 1\right\}$ and let $\Lambda$ be processes with sample paths in $\mathscr{M}([0, \infty))$ such that $\theta_{n} \Rightarrow \Lambda$. Assume that $Y_{n}$ is independent of $\theta_{n}$ for all $n$. Then $Y_{n} \circ \theta_{n} \Rightarrow Y \circ \Lambda$.

P r o o f. For $y \in \mathscr{M}([0, \infty))$ let $D(y):=\{t \geqslant 0: y(t-0) \neq y(t)\}$. Since $\Lambda$ is strictly increasing and $Y$ is stochastically continuous, $D(Y) \cap$ $D(\Lambda)=\varnothing$ a.s. Hence by Theorem 3 in [14],

$$
\left\{\widetilde{Y}_{n}(t)=Y_{n}\left(\theta_{n}(t)\right): t>0\right\} \Longrightarrow\{\tilde{Y}(t)=Y(\Lambda(t)): t>0\} .
$$

Proposition 2.1 is proved.

At the end of this section we consider the i.i.d. case and give a Functional Transfer Theorem (FTT) in analogy to the famous Gnedenko-Fakhim Transfer Theorem (cf. [6], [5]). But let us first see the meaning of weak convergence $\theta_{n}=\tau_{n}^{-1} \circ \theta \circ \tau_{n} \Rightarrow \Lambda$ by considering three examples.

$\mathrm{Ex}$ a m ple 2. Let $\tau_{n}(t)=n t$ and $\theta(t)=c t^{a}, 0<a<1$. Then $\theta_{n}=c t^{a} / n^{1-a} \rightarrow 0, n \rightarrow \infty$.

$\mathrm{Ex} \mathrm{a} \mathrm{m} \mathrm{ple} 3$. Let $\tau_{n}(t)=n t$ and $\theta(t)=e^{t}$. Then $\theta_{n}(t)=e^{n t} / n \rightarrow$ $\infty, n \rightarrow \infty$.

$\mathrm{E} \times \mathrm{a} \mathrm{m} \mathrm{p} \mathrm{le} 4$. Let $\theta$ possess the scaling property: for all $t>0$ there exists a subsequence $m_{n}=[n t]$ such that for $n \rightarrow \infty$

$$
\theta \circ \tau_{n}(t) \sim \tau_{[n t]} \circ \theta(1)
$$


Then, using the regularity of $\left\{\tau_{n}\right\}$ (i.e., $\tau_{n}^{-1} \circ \tau_{[n t]}$ converges pointwise to a continuous strictly increasing mapping $\left.\sigma_{t}:[0, \infty) \rightarrow[0, \infty)\right)$ we get

$$
\tau_{n}^{-1} \circ \theta \circ \tau_{n}(t) \sim \tau_{n}^{-1} \circ \tau_{[n t]} \circ \theta(1) \Longrightarrow \sigma_{t} \circ \theta(1)=\Lambda(t) .
$$

Obviously in this case $\Lambda(t)$ is continuous and increasing in $t$ but does not have independent increments.

Thus, we see that $\tau_{n}$ and $\theta$ must have «comparable» behavior at infinity in order for $\Lambda$ to be finite and nondegenerate.

Theorem 2.1 (FTT). Let $\widetilde{\mathscr{N}}=\left\{\left(T_{k}, X_{k}\right)\right\}$ and $\mathscr{N}=\left\{\left(t_{k}, X_{k}\right)\right\}$ be Bernoulli point processes with counting functions $N(t)$ and $k(t)$, respectively. Let $\left\{X_{k}\right\}$ be i.i.d. random variables and $\xi_{n}(t, x)=\left(\tau_{n}(t), u_{n}(x)\right)$ a regular norming sequence. Suppose there is a stochastically continuous time process $\theta$ in $\mathscr{M}([0, \infty))$ independent of $\left\{X_{k}\right\}$, satisfying (2.5), and such that $N(t)=k(\theta(t))$. Denote the distribution function of $\theta(1)$ by $Q$, and set $N\left(\tau_{n}(t)\right)=N_{n}(t)$ and $k\left(\tau_{n}(t)\right)=k_{n}(t)$. Assume further the weak convergence as $n \rightarrow \infty$

$$
\mathbf{P}\left\{\bigvee_{k=1}^{k_{n}(t)} u_{n}^{-1}\left(X_{k}\right)<x\right\} \longrightarrow f(t, x):=\mathbf{P}\{Y(t)<x\}
$$

Then

$$
\tilde{Y}_{n}(t)=\bigvee_{k=1}^{N_{n}(t)} u_{n}^{-1}\left(X_{k}\right) \Longrightarrow \tilde{Y}(t)=Y(\Lambda(t))
$$

and there exists a time change $\tau(s)$ such that

$$
\mathbf{P}\{\tilde{Y}(t)<x\}=\int_{0}^{\infty} f^{\tau(s)}(1, x) d Q\left(\sigma_{t}^{-1}(s)\right)=\mathbf{E}[f(1, x)]^{\tau \circ \Lambda(t)} .
$$

Here $\sigma_{t}$ is the time change from (2.6).

$\mathrm{Pr}$ o of. Let $Y_{n}$ be the extremal process associated with

$$
\mathscr{N}_{n}=\left\{\left(\tau_{n}^{-1}\left(t_{k}\right), u_{n}^{-1}\left(X_{k}\right)\right): k \geqslant 1\right\},
$$

and let $F$ be the distribution function of $X_{1}$. By assumption

$$
\begin{aligned}
f_{n}(t, x) & :=\mathbf{P}\left\{Y_{n}(t)<x\right\}=\mathbf{P}\left\{\bigvee_{k=1}^{k_{n}(t)} X_{k}<u_{n}(x)\right\} \\
& =F^{k_{n}(t)}\left(u_{n}(x)\right) \longrightarrow f(t, x) .
\end{aligned}
$$

On the other hand, since

$$
f_{n}(t, x)=\left[F^{k_{n}(1)}\left(u_{n}(x)\right)\right]^{k_{n}(t) / k_{n}(1)}
$$

and since $f(1, x) \in(0,1)$, we conclude that for all $t>0$ there exists (perhaps up to a subsequence)

$$
\lim _{n} \frac{k_{n}(t)}{k_{n}(1)}=: \tau(t),
$$


hence $f(t, x)=f^{\tau(t)}(1, x)$. The limit extremal process $Y$ is stochastically continuous. Note that the weak convergence of the univariate marginals entails $Y_{n} \Rightarrow Y$, in view of Theorem 6.1 in [2]. Now let us express the partial maxima $\widetilde{Y}_{n}$ as

$$
\tilde{Y}_{n}(t)=\bigvee_{k=1}^{N_{n}(t)} u_{n}^{-1}\left(X_{k}\right)=\bigvee_{k=1}^{k_{n}\left(\theta_{n}(t)\right)} u_{n}^{-1}\left(X_{k}\right)=Y_{n} \circ \theta_{n}(t) .
$$

One can see that the conditions of Proposition 2.1 are satisfied. Applying Proposition 2.1 and (2.5) we have

$$
Y_{n} \circ \theta_{n} \Longrightarrow Y \circ \Lambda=: \tilde{Y}
$$

and furthermore

$$
\begin{aligned}
\mathbf{P}\{Y(\Lambda(t))<x\} & =\int_{0}^{\infty} f(s, x) d \mathbf{P}\{\Lambda(t)<s\} \\
& =\int_{0}^{\infty} f^{\tau(s)}(1, x) d Q\left(\sigma_{t}^{-1}(s)\right) .
\end{aligned}
$$

Theorem 2.1 is proved.

$\mathrm{R}$ e $\mathrm{m}$ a r k 1. Note that (2.7) is a consequence of the weak convergence $Y_{n} \Rightarrow Y$. If we do suppose (2.7), then we need only assume $Y_{n}(1) \stackrel{\mathrm{d}}{\longrightarrow} Y(1)$ instead of $Y_{n} \Rightarrow Y$ in order to get $\tilde{Y}_{n} \Rightarrow \tilde{Y}$.

$\mathrm{R}$ e $\mathrm{mark} 2$. From the proof one can see that FTT remains true if condition (2.5) is replaced by the more general convergence condition $\theta_{n} \Rightarrow \Lambda$ of Proposition 2.1. In this case

$$
\mathbf{P}\left\{\bigvee_{k=1}^{N_{n}(t)} u_{n}^{-1}\left(X_{k}\right)<x\right\} \longrightarrow \int_{0}^{\infty} f^{\tau(s)}(1, x) d Q_{t}(s),
$$

where $Q_{t}(s):=\mathbf{P}\{\Lambda(t)<s\}$.

$\mathrm{R}$ e $\mathrm{m}$ a r k 3 . One can see also that the FTT remains true if $X_{k}$ are assumed independent but not identically distributed. Then

$$
\mathbf{P}\left\{\bigvee_{k=1}^{N_{n}(t)} u_{n}^{-1}\left(X_{k}\right)<x\right\} \longrightarrow \int_{0}^{\infty} f(s, x) d Q_{t}(s)=\mathbf{E} f(\Lambda(t), x) .
$$

Corollary 1. Let $\tau_{n}^{-1} \circ \theta \circ \tau_{n} \Rightarrow \Lambda$. Then the time process $\Lambda$ satisfies $\sigma_{s} \circ \Lambda \circ \sigma_{s}^{-1}(t) \stackrel{\mathrm{d}}{=} \Lambda(t)$ for all $s>0$; hence $\Lambda$ is stochastically continuous.

$\mathrm{P}$ r o o f. Let us assume that there is a $t_{0}>0$ such that $\Lambda\left(t_{0}-0\right)<$ $\Lambda\left(t_{0}\right)$. We can choose $s>0$ so that $\sigma_{s}^{-1}\left(t_{0}\right)$ is a continuity point of $\Lambda$. Hence

$$
\Lambda\left(t_{0}\right) \stackrel{\mathrm{d}}{=} \sigma_{s} \circ \Lambda \circ \sigma_{s}^{-1}\left(t_{0}\right) \stackrel{\text { a.s. }}{=} \sigma_{s} \circ \Lambda \circ \sigma_{s}^{-1}\left(t_{0}-0\right) \stackrel{\mathrm{d}}{=} \Lambda\left(t_{0}-0\right),
$$

which contradicts the above assumption. 
Corollary 2. The limit process $\tilde{Y}$ in (2.8) has the properties:

i) it is stochastically continuous;

ii) it is self-similar with respect to $\mathscr{L}=\left\{\left(\sigma_{s}, U_{s}\right): s>0\right\}$;

iii) it does not have stationary increments;

iv) it is not max-stable.

P r o o f. i) $\tilde{Y}$ is composition of two stochastically continuous processes.

ii) We have to show that $\tilde{Y}\left(\sigma_{s}(t)\right) \stackrel{\text { d }}{=} U_{s} \circ \tilde{Y}(t)$ for all $s>0$. For a fixed $s>0$, we have $N_{[n s]}(t)=N\left(\tau_{[n s]}(t)\right)=N_{n}\left(\tau_{n}^{-1} \circ \tau_{[n s]}(t)\right)$ and by the FTT and continuity of the composition we conclude the weak convergence

$$
\bigvee_{k=1}^{N_{[n s]}(t)} u_{n}^{-1}\left(X_{k}\right) \Longrightarrow \tilde{Y}\left(\sigma_{s}(t)\right)
$$

On the other hand,

$$
\bigvee_{k=1}^{N_{[n s]}(t)} u_{n}^{-1}\left(X_{k}\right)=u_{n}^{-1} \circ u_{[n s]}\left(\bigvee_{k=1}^{N_{[n s]}(t)} u_{[n s]}^{-1}\left(X_{k}\right)\right) \Longrightarrow U_{s} \circ \tilde{Y}(t)
$$

which entails the self-similarity of $\tilde{Y}$.

iii) and iv) are easily seen from the right-hand side of (2.8). Corollary 2 is proved.

In [10, Propositions 2.1 and 2.3], it is shown that the limit extremal process $Y$ (respectively, distribution function $f$ ) is self-similar. So we refer to the process $\tilde{Y}(t)=Y(\Lambda(t))$ as random time-changed or compound selfsimilar extremal process. In the next section we study its properties.

3. Properties of a compound extremal process. In this section we consider the composition $\tilde{Y}=Y \circ \Lambda, \tilde{Y}:[0, \infty) \rightarrow[0, \infty)^{d}$, of an extremal process $Y:[0, \infty) \rightarrow[0, \infty)^{d}, Y(0)=C(0)=0$ a.s., and a stochastically continuous time-process $\Lambda:[0, \infty) \rightarrow[0, \infty)$ independent of $Y$ and with a.a. sample paths in the functional space $\mathscr{M}([0, \infty))$.

In general, the compound extremal process $Y \circ \Lambda$ may have dependent max-increments, cf. Example 4 of the previous section.

Property 3.1. Let $Y$ be self-similar extremal process with respect to the norming group $\left\{\eta_{s}(t, x)=\left(\sigma_{s}(t), U_{s}(x)\right): s>0\right\}$, i.e., $L_{s} \circ Y(t)=Y \circ \sigma_{s}(t)$, and let the random time change be of the form $\Lambda(t)=\sigma_{t}(\theta)$, where $\theta$ is a positive random variable. Then the compound extremal process is self-similar with respect to the group $\left\{\eta_{s}^{*}(t, x)=\left(t s, U_{s}(x)\right): s>0\right\}$.

P r o o f. Indeed,

$$
\begin{aligned}
U_{s} \circ \tilde{Y}(t) & =U_{s} \circ Y(\Lambda(t))=Y \circ \sigma_{s}(\Lambda(t))=Y \circ \sigma_{s}\left(\sigma_{t}(\theta)\right) \\
& =Y \circ \sigma_{s t}(\theta)=Y \circ \Lambda(s t)=\tilde{Y}(s t) .
\end{aligned}
$$


The first question naturally arising in our framework is: «under what conditions on $\Lambda$ and $Y$ is the composition $Y \circ \Lambda$ an extremal process in the sense of (1.1)».

Denote by

$$
\mathscr{N}_{0}=\left\{\left(\Gamma_{k}, Z_{k}\right): k \geqslant 1\right\}
$$

the Bernoulli point process associated with $Y$. Let $U_{Y}(s, t]$ (respectively, $\left.U_{\widetilde{Y}}(s, t]\right)$ be the max-increment of $Y$ (respectively, of $\widetilde{Y}$ ) over a time interval $(s, t]$. Then

$$
\begin{aligned}
& U_{\widetilde{Y}}(0, s]=\left\{\bigvee Z_{k}: 0<\Gamma_{k} \leqslant \Lambda(s)\right\}, \\
& U_{\widetilde{Y}}(s, t]=\left\{\bigvee Z_{k}: \Lambda(s)<\Gamma_{k} \leqslant \Lambda(t)\right\} .
\end{aligned}
$$

The intervals $(0, \Lambda(s)]$ and $(\Lambda(s), \Lambda(t)]$ are a.s. disjoint since the timeprocess $\Lambda$ has a.s. strictly increasing sample paths.

Theorem 3.1. Assume that $Y$ is an extremal process and $\Lambda$ is a timeprocess independent of $Y$ and with a.a. sample paths in $\mathscr{M}([0, \infty))$. In this framework the compound process $Y \circ \Lambda$ is an extremal process if and only if

i) $\Lambda$ has independent additive increments,

ii) $Y$ has homogeneous max-increments.

P r o o f. 1. Sufficiency. In view of (1.1) we have to check that

a) $\tilde{Y}$ has right-continuous increasing sample paths,

b) for arbitrary $s>0, t>s$, the random vectors $U_{\widetilde{Y}}(0, s]$ and $U_{\widetilde{Y}}(s, t]$ are independent.

In our framework condition a) is obviously satisfied. Recall that the lower curve of an extremal process with homogeneous increments is constant. Thus we may assume for the lower curve $C$ of $Y$ that $C(t) \equiv 0$.

Let us consider in detail the probability $\mathbf{P}\left\{U_{\widetilde{Y}}(0, s]<x, U_{\widetilde{Y}}(s, t]<y\right\}$ for $0<s<t$ and arbitrary $x, y \in[0, \infty)^{d}$. Using step by step the assumptions: $Y$ and $\Lambda$ are independent, $Y$ has independent max-increments, $\Lambda$ has independent increments, and $Y$ has homogeneous increments, we get:

$$
\begin{gathered}
\mathbf{P}\left\{U_{\widetilde{Y}}(0, s]<x, U_{\widetilde{Y}}(s, t]<y\right\} \\
=\iint_{\{(p, q): 0<p<q\}} \mathbf{P}\left\{U_{Y}(0, \Lambda(s)]<x, U_{Y}(\Lambda(s), \Lambda(t)]<y \mid \Lambda(s)=p,\right. \\
\Lambda(t)=q\} d \mathbf{P}\{\Lambda(s)<p, \Lambda(t)<q\} \\
=\iint_{\{(p, q): 0<p<q\}} \mathbf{P}\left\{U_{Y}(0, p]<x\right\} \\
=\int_{0}^{\infty} \int_{0}^{\infty} \mathbf{P}\left\{U_{Y}(0, p]<x\right\} \\
\times \mathbf{P}\left\{U_{Y}(p, q]<y\right\} d \mathbf{P}\{\Lambda(s)<p, \Lambda(t)<q\} \\
\quad \mathbf{P}\left\{U_{Y}(p, p+h]<y\right\} d \mathbf{P}\{\Lambda(s)<p\} d \mathbf{P}\{\Lambda(t)-\Lambda(s)<h\}
\end{gathered}
$$




$$
\begin{aligned}
= & \int_{0}^{\infty} \mathbf{P}\left\{U_{Y}(0, p]<x\right\} d \mathbf{P}\{\Lambda(s)<p\} \\
& \times \int_{0}^{\infty} \mathbf{P}\left\{U_{Y}(0, h]<y\right\} d \mathbf{P}\{\Lambda(t)-\Lambda(s)<h\} \\
= & \mathbf{P}\left\{U_{\widetilde{Y}}(0, s]<x\right\} \mathbf{P}\left\{U_{Y}(0, \Lambda(t)-\Lambda(s)]<y\right\} \\
= & \mathbf{P}\left\{U_{\widetilde{Y}}(0, s]<x\right\} \mathbf{P}\left\{U_{Y}(\Lambda(s), \Lambda(t)]<y\right\} \\
= & \mathbf{P}\left\{U_{\widetilde{Y}}(0, s]<x\right\} \mathbf{P}\left\{U_{\widetilde{Y}}(s, t]<y\right\} .
\end{aligned}
$$

2. Necessity. Now we assume that the composition $\tilde{Y}=Y \circ \Lambda$ is an extremal process. Then, necessarily, $\Lambda$ has independent increments, i.e., $\Lambda(s) \perp \Lambda(t)-\Lambda(s)$ for all $0 \leqslant s<t$. We have to show only that $Y$ has homogeneous increments, or equivalently that the counting measure $N$ of the associated Bernoulli point process $\mathscr{N}_{0}$ is homogeneous.

Indeed, the independence $U_{\widetilde{Y}}(0, s] \perp U_{\widetilde{Y}}(s, t]$ implies

$$
\bigvee_{0}^{N(\Lambda(s))} Z_{k} \perp \bigvee_{N(\Lambda(s))+1}^{N(\Lambda(t))} Z_{k}
$$

which implies

$$
N(0, \Lambda(s)] \perp N(\Lambda(s), \Lambda(s)-(\Lambda(t)-\Lambda(s))] .
$$

This is possible only if $N(\Lambda(s), \Lambda(t)$ ] does not depend on $\Lambda(s)$ but on $\Lambda(t)-\Lambda(s)$ only. For the counting measure $N$ this means that $N$ is homogeneous in the sense that $N(s, t] \stackrel{\mathrm{d}}{=} N(0, t-s]$. Theorem 3.1 is proved.

Let us now simplify the model and assume additionally that $Y$ is a self-similar extremal process with homogeneous max-increments. Then the univariate marginal distribution function $f_{t}(x)=\mathbf{P}\{Y(t)<x\}$ is max-stable and satisfies

$$
f_{t}(x)=f_{1}^{t}(x)=f_{1}\left(U_{t}^{-1}(x)\right) \quad \forall t>0
$$

(cf. [10]). Without loss of generality we may and do assume that $f_{1}(x)$ has Fréchet marginals, i.e., $f_{1}\left(x_{i}\right)=e^{-x_{i}^{-\alpha}}, \alpha>0, i=1, \ldots, d$. So $f_{t}(x)=$ $\exp \left\{-t \nu_{\alpha}\left(A_{x}^{c}\right)\right\}$. Here $\nu_{\alpha}$ is the exponent measure of $Y(1)$ and $A_{x}^{c}=\{y \in$ $\left.\mathbf{R}_{+}^{d}: y<x\right\}^{c}$. The exponent measure $\nu_{\alpha}$ bears the dependence structure of $Y(1)$, see, e.g., [9]. Now observe that

$$
s^{H} Y(t)=Y(s t), \quad \forall t>0, \quad s>0, \quad H=\frac{1}{\alpha},
$$

i.e., $Y(t)$ is self-similar with respect to the multiplicative group $\left\{\eta_{s}(t, x)=\right.$ $\left.\left(s t, s^{H} x\right): s>0\right\}$.

Property 3.2. Denote the Laplace transform of the time process $\Lambda(t)$ by $l_{t}(r)=\operatorname{E} \exp \{-\Lambda(t) r\}, r>0$, and its distribution function by 
$\mathbf{P}\{\Lambda(t)<s\}=G_{t}(s)$. The compound extremal process is then distributed by $\mathbf{P}\{\tilde{Y}(t)<x\}=l_{t}\left(\nu_{\alpha}\left(A_{x}^{c}\right)\right)$.

Indeed,

$$
\begin{aligned}
\mathbf{P}\{\tilde{Y}(t)<x\} & =\int_{0}^{\infty} \mathbf{P}\{Y(s)<x \mid \Lambda(t)=s\} d G_{t}(s)=\int_{0}^{\infty} f_{1}^{s}(x) d G_{t}(s) \\
& =\mathbf{E} \exp \left\{-\Lambda(t) \nu_{\alpha}\left(A_{x}^{c}\right)\right\}=l_{t}\left(\nu_{\alpha}\left(A_{x}^{c}\right)\right) .
\end{aligned}
$$

Property 3.3. Assume $\Lambda(t)$ has independent increments. Then the compound process $\tilde{Y}$ is max-id with mean measure

$$
\tilde{\mu}\left([0, t] \times A_{x}^{c}\right)=\int_{0}^{\infty}\left(1-e^{-u \nu_{\alpha}\left(A_{x}^{c}\right)}\right) d \mathscr{L}_{t}(u), \quad x>0,
$$

where $\mathscr{L}_{t}$ is the Lévy measure of $\Lambda(t)$.

$\mathrm{P}$ r o o f. As a stochastically continuous process with independent increments the time process $\Lambda$ is infinitely divisible. Since $\Lambda(t)$ is positive and increasing, its characteristic function $\phi_{t}$ has the form

$$
\phi_{t}(s)=\exp \left\{\int_{0}^{\infty}\left(e^{i s u}-1\right) d \mathscr{L}_{t}(u)\right\}
$$

By Property 3.2, and since $l_{t}(s)=\phi_{t}(i s)$, we can further write:

$$
\begin{aligned}
-\ln \mathbf{P}\{\tilde{Y}(t)<x\} & =-\ln l_{t}\left(\nu_{\alpha}\left(A_{x}^{c}\right)\right)=-\ln \phi_{t}\left(-i \ln f_{1}(x)\right) \\
& =\int_{0}^{\infty}\left(1-e^{-u \nu_{\alpha}\left(A_{x}^{c}\right)}\right) d \mathscr{L}_{t}(u) .
\end{aligned}
$$

The measure $\tilde{\mu}$ defined by

$$
\widetilde{\mu}\left([0, t] \times A_{x}^{c}\right):=\int_{0}^{\infty}\left(1-e^{-u \nu_{\alpha}\left(A_{x}^{c}\right)}\right) d \mathscr{L}_{t}(u)
$$

has all the properties of an exponent measure on $[0, \infty)^{1+d}$ (cf. [1], [9]). Since $\mathbf{P}\{\tilde{Y}(t)<x\}=\exp \left\{-\tilde{\mu}\left([0, t] \times A_{x}^{c}\right)\right\}$, the compound extremal process is max-id. Property 3.3 is proved.

In the case when $\Lambda$ has homogeneous increments, i.e.,

$$
\tilde{\mu}\left([0, t] \times A_{x}^{c}\right)=t \int_{0}^{\infty}\left(1-e^{-u \nu_{\alpha}\left(A_{x}^{c}\right)}\right) d \mathscr{L}_{1}(u)
$$

one gets the following asymptotic for $t \rightarrow 0$ and for $x$ far away from 0 as a by-product of the proof:

$$
\lim _{t \rightarrow 0} \frac{1}{t} \mathbf{P}\left\{\tilde{Y}(t) \in A_{x}^{c}\right\} \sim \int_{0}^{\infty}\left(1-e^{-u \nu_{\alpha}\left(A_{x}^{c}\right)}\right) d \mathscr{L}_{1}(u), \quad x \rightarrow \infty .
$$

For any Borel set $B \subset[0, \infty)^{d}$ and $x \in(0, \infty)^{d}$ let $B / x$ denote the set $\{s \in[0, \infty): s x \in B\}$. Define the measure $Q_{t}$ on $[0, \infty)$ corresponding to the 
distribution function $G_{t}$ of $\Lambda(t)$ by $Q_{t}(A):=\int_{0}^{\infty} I_{A}(s) d G_{t}(s), A \subset[0, \infty)$, and put $T_{H} Q_{t}(A):=Q_{t}\left(\left\{s^{H}: s \in A\right\}\right)$. Then the distribution function of the compound process can be expressed as follows (cf. [7]).

Property 3.4. $\mathbf{P}\{\tilde{Y}(t)<x\}=\mathbf{E}\left(T_{H} Q_{t}\right)\left(A_{x} / Y(1)\right)$.

P r o o f. For $x \in(0, \infty)^{d}$ we have

$$
\begin{aligned}
\mathbf{P}\{\tilde{Y}(t)<x\} & =\mathbf{P}\left\{\Lambda^{H}(t) Y(1) \in A_{x}\right\}=\int \mathbf{P}\left\{\Lambda^{H}(t) \in \frac{A_{x}}{y}\right\} d f_{1}(y) \\
& =\int d f_{1}(y) \int_{0}^{\infty} I_{A_{x} / y}\left(s^{H}\right) G_{t}(d s)=\mathbf{E}\left(T_{H} Q_{t}\left(\frac{A_{x}}{Y(1)}\right)\right),
\end{aligned}
$$

where the integral is taken over $[0, \infty)^{d} \backslash\{0\}$. Property 3.4 is proved.

$\mathrm{R} \mathrm{e} \mathrm{m}$ a r k. By Corollary 1 in the previous section if $\tau_{n}^{-1} \circ \theta \circ \tau_{n} \Rightarrow \Lambda$ then $\sigma_{s} \circ \Lambda \circ \sigma_{s}^{-1}(t) \stackrel{d}{=} \Lambda(t)$ for all $s>0$. For $\sigma_{s}^{-1}(t)=s t$ the latter equation means

$$
\Lambda(t) \stackrel{d}{=} t \Lambda(1) .
$$

In this section we do not assume the above limit relation. Yet (3.1) has to hold if both processes $\tilde{Y}$ and $Y$ are assumed self-similar with respect to the same multiplicative group $\mathscr{L}=\left\{\eta_{s}(t, x)=\left(s t, s^{H} x\right): s>0\right\}$.

Property 3.5. a) If $\tilde{Y}$ and $Y$ are self-similar with respect to the multiplicative group $\mathscr{L}$, then $\Lambda$ has stationary increments.

b) If (3.1) holds and if $Y$ is self-similar with respect to $\mathscr{L}$, then $\tilde{Y}$ is also self-similar with respect to the same $\mathscr{L}$.

P r o o f. a) Indeed, one can check that both

$$
\tilde{Y}(t) \stackrel{d}{=} Y(\Lambda(t)) \stackrel{d}{=} \Lambda^{H}(t) Y(1) \quad \text { and } \quad \tilde{Y}(t) \stackrel{d}{=} t^{H} \tilde{Y}(1)
$$

entail (3.1), or equivalently $\Lambda(t+h) \stackrel{\mathrm{d}}{=} \Lambda(t)+\Lambda(h)$, i.e., $\Lambda$ has stationary increments.

b) Indeed,

$$
\tilde{Y}(s t)=Y(\Lambda(s t)) \stackrel{\mathrm{d}}{=} Y(s \Lambda(t)) \stackrel{\mathrm{d}}{=} s^{H} \tilde{Y}(t) .
$$

Property 3.5 is proved.

Finally, note that the compound extremal process $\tilde{Y}$ considered in this section can be decomposed in a product of two independent random processes, namely

$$
\tilde{Y}(t)=Y(\Lambda(t))=\Lambda^{H}(t) Y(1)=\left(\Lambda^{H}(t) t^{-H}\right)\left(t^{H} Y(1)\right)=M(t) Y(t),
$$

where $M(t):=(\Lambda(t) / t)^{H}$. So, the stability character of $\tilde{Y}$ is governed by the self-similar process $Y(t)$ and the volatility of $\widetilde{Y}$ is borne by the random time $M(t)$. 
4. Application to ruin probability. The basic Bernoulli point process $\mathscr{N}=\left\{\left(T_{k}, X_{k}\right): k \geqslant 1\right\}$, we are dealing with here, can be interpreted as describing a particular insurance model with

a) claim size process: the claim sizes $\left\{X_{k}\right\}$ are positive i.i.d. random variables whose distribution function $F$ has a regularly varying tail, namely $\bar{F} \in R V_{-\alpha}$. We consider the «very heavy tail case» $0<\alpha<1$ when $\mathbf{E} X=\infty$.

Activity rates with very heavy tails are studied in [8]. In fact, investigations in the very heavy tail case are not only of a theoretical interest but they are useful in connection with modeling catastrophic events and reinsurance problems.

b) claim times: the claims occur at times $\left\{T_{k}\right\}$, where $T_{1}<T_{2}<\cdots$, $T_{k} \rightarrow \infty$ a.s., and the number of claims in the interval $[0, t], N(t)=$ $\sum_{k} I_{[0, t]}\left(T_{k}\right)$, satisfies the condition (2.1), i.e., there exists a time process $\theta:[0, \infty) \rightarrow[0, \infty)$ such that $N(t)=k(\theta(t))$, where $k$ is deterministic counting function whose asymptotic property we specify below;

c) both sequences $\left\{X_{k}\right\}$ and $\left\{T_{k}\right\}$ are independent.

With the point process $\mathscr{N}$ we associate three random processes:

(i) the accumulated claim process $S(t)=\sum_{k=1}^{N(t)} X_{k}$;

(ii) the extremal claim process $Y(t)=\bigvee_{k=1}^{N(t)} X_{k}$;

(iii) the risk process $R(t)=c(t)-S(t)$, where $u:=c(0) \geqslant 0$ is the initial capital and $c(t)$ is the premium income up to time $t$ (hence it is an increasing curve); we assume $c(t)$ right-continuous.

In order to estimate the ruin probability in our framework we follow the idea of a stable Lévy motion approximation of the risk process $R(t)$ developed in [4]. To this end we transform time and space properly and get a sequence of risk processes weakly convergent to a risk process whose accumulated claim process is an $\alpha$-stable Lévy motion.

Let $\xi_{n}(t, x)=\left(\tau_{n}(t), u_{n}(x)\right)$ be a norming sequence of time-space changes and let $L(\cdot)$ be a slowly varying function. We suppose that $\tau_{n}(t)$ satisfy the following condition:

d) $k_{n}(t) / k_{n} \rightarrow t$ with $k_{n}(t)=k\left(\tau_{n}(t)\right)$ and $k_{n}:=k_{n}(1)$.

The choice of $u_{n}(x)=k_{n}^{1 / \alpha} L\left(k_{n}\right) x$ is determined by the regularly varying tail of the claim size distribution function $F$. Denote $\tau_{n}^{-1}\left(T_{k}\right)=: T_{n k}$ and $u_{n}^{-1}\left(X_{k}\right)=X_{k} /\left(k_{n}^{1 / \alpha} L\left(k_{n}\right)\right)=: X_{n k}$. Now, the sequence of point processes

$$
\mathscr{N}_{n}=\left\{\left(T_{n k}, X_{n k}\right): k \geqslant 1\right\}
$$

generates associated sequences of:

(i) the counting functions $N_{n}(t)=k_{n}\left(\theta_{n}(t)\right)$, where as before $\theta_{n}=$ $\tau_{n}^{-1} \circ \theta \circ \tau_{n}$

(ii) the accumulated claims $\sum_{k=1}^{N_{n}(t)} X_{n k}=S_{n}\left(\theta_{n}(t)\right)$; here $S_{n}(t)$ is an abbreviation for $\sum_{k=1}^{k_{n}(t)} X_{n k}$; 
(iii) the extremal claims $\bigvee_{k=1}^{N_{n}(t)} X_{n k}=Y_{n}\left(\theta_{n}(t)\right)$, where $Y_{n}(t)$ is the extremal process $\bigvee_{k=1}^{k_{n}(t)} X_{n k}$

(iv) the risk process $R_{n}(t)=c^{(n)}(t)-S_{n}\left(\theta_{n}(t)\right)$, where $c^{(n)}(t)=u_{n}^{-1} \circ$ $c \circ \tau_{n}(t)$.

In our insurance model described by a)-d) let us assume additionally that

e) $\theta_{n} \Rightarrow \Lambda, \Lambda$ in $\mathscr{M}([0, \infty))$.

In fact the last two assumptions are implicit conditions on the claim times process $\left\{T_{k}\right\}$ (through $\theta(\cdot)$ ), e.g., they imply that

$$
k_{n}^{-1} N_{n}(t) \longrightarrow \Lambda(t), \quad n \rightarrow \infty .
$$

The following examples illustrate the impact of d) for the time change $\tau_{n}(t)$.

$\mathrm{Ex}$ a $\mathrm{m} \mathrm{ple} \mathrm{5.} \mathrm{For} \mathrm{large} n$
a) $k(t)=[a t+b] \quad$ implies $\tau_{n}(t)=\frac{k_{n} t-b}{a}$;
b) $k(t)=[\ln t] \quad$ implies $\tau_{n}(t)=e^{k_{n} t}$;
c) $k(t)=\left[a^{t}\right] \quad$ implies $\tau_{n}(t)=\frac{\ln k_{n} t}{\ln a}$.

Assume that $\sum_{k=1}^{k_{n}} X_{n k}$ converges to an $\alpha$-stable random variable $Z_{\alpha}$. Using the stable FCLT for sum and maxima of positive i.i.d. r.v.'s one can see that conditions a)-d) imply the convergences

$$
S_{n}(t)=\sum_{k=1}^{k_{n}(t)} X_{n k} \Longrightarrow Z_{\alpha}(t), \quad Z_{\alpha}(1)=Z_{\alpha}
$$

where $Z_{\alpha}(t)$ is a one-sided $\alpha$-stable Lévy motion, and

$$
Y_{n}(t)=\bigvee_{k=1}^{k_{n}(t)} X_{n k} \Longrightarrow Y_{\alpha}(t)
$$

where the univariate marginals of the limit extremal process are Fréchet distributed, i.e.,

$$
\mathbf{P}\left\{Y_{\alpha}(t)<x\right\}=\Phi_{\alpha}^{t}(x)=\exp \left\{-t x^{-\alpha}\right\}, \quad x \geqslant 0, \quad t \geqslant 0 .
$$

Let us observe that the conditions of Proposition 2.1 are satisfied, hence we conclude

$$
Y_{n} \circ \theta_{n} \Rightarrow Y_{\alpha} \circ \Lambda, \quad S_{n} \circ \theta_{n} \Rightarrow Z_{\alpha} \circ \Lambda
$$

By Proposition 3.21 in [9] convergence (4.2) is equivalent to the weak convergence of the associated point processes. Denote the limit Poisson point process by $\mathscr{N}_{0}$, say $\mathscr{N}_{0}=\left\{\left(\Gamma_{k}, Z_{k}\right): k \geqslant 1\right\}$. Its mean measure is

$$
\mu_{0}([0, t] \times[x, \infty))=t\left(-\ln \Phi_{\alpha}(x)\right)=t x^{-\alpha} .
$$


Moreover, since the time process $\Lambda$ is independent of the space points $\left\{Z_{k}\right\}, \Lambda$ is independent of $Y_{\alpha}$ and $Z_{\alpha}$.

Let us come back to the sequence of the risk processes $R_{n}(t)$. To reach the weak convergence

$$
R_{n}(t)=c^{(n)}(t)-S_{n}\left(\theta_{n}(t)\right) \Longrightarrow c_{0}(t)-Z_{\alpha}(\Lambda(t))=: R_{\alpha}(t)
$$

we need also the asymptotic relation:

f) $c^{(n)} \stackrel{w}{\rightarrow} c_{0}, n \rightarrow \infty, c_{0}$ increasing curve with $c_{0}(0)>0$.

Note that condition $\mathrm{f}$ ) plays the role of a «safety loading condition in the very heavy tail case» since it guaranties a positive probability to survive (see (4.4) below).

Now we are ready to obtain lower and upper bounds of the ruin probability associated with the limit risk process $R_{\alpha}(t)$. Below we make use of the self-similarity of $Z_{\alpha}$ and of the reflection principle proved in [4, Theorem 5]. Denote $Q_{t}(s):=\mathbf{P}\{\Lambda(t)<s\}, G_{\alpha}(y):=\mathbf{P}\left\{Z_{\alpha}(1)<y\right\}, u_{0}:=c_{0}(0)$. Note that the constant $\rho:=\mathbf{P}\left\{Z_{\alpha}(1)>0\right\}$ from [4, Theorem 5] is equal to 1 here. We have

$$
\begin{aligned}
\psi\left(u_{0}, t\right) & =\mathbf{P}\left\{\inf _{0 \leqslant s \leqslant t} R_{\alpha}(s)<0\right\} \\
& =\mathbf{P}\left\{\sup _{0 \leqslant s \leqslant t}\left\{\left[\sum Z_{k}: \Gamma_{k} \leqslant \Lambda(s)\right]-c_{0}(s)\right\}>0\right\} \\
& \leqslant \mathbf{P}\left\{\sup _{0 \leqslant s \leqslant t} Z_{\alpha}(\Lambda(s))>u_{0}\right\} \leqslant \mathbf{P}\left\{\sup _{0 \leqslant s \leqslant \Lambda(t)} Z_{\alpha}(s)>u_{0}\right\} \\
& =\int_{0}^{\infty} \mathbf{P}\left\{\sup _{0 \leqslant s \leqslant v} Z_{\alpha}(s)>u_{0}\right\} d Q_{t}(v) \\
& \leqslant \int_{0}^{\infty} \mathbf{P}\left\{v^{1 / \alpha} Z_{\alpha}(1)>u_{0}\right\} d Q_{t}(v)=\mathbf{P}\left\{\Lambda^{1 / \alpha}(t) Z_{\alpha}(1)>u_{0}\right\} \\
& =\int_{0}^{\infty} \bar{Q}_{t}\left(\left(\frac{u_{0}}{y}\right)^{\alpha}\right) d G_{\alpha}(y)=: \bar{\psi}\left(u_{0}, t\right) .
\end{aligned}
$$

Here $\bar{Q}_{t}=1-Q_{t}$. On the other hand,

$$
\begin{aligned}
\psi\left(u_{0}, t\right) & \geqslant \mathbf{P}\left\{\sup _{0 \leqslant s \leqslant t}\left\{\left[\vee Z_{k}: \Gamma_{k} \leqslant \Lambda(s)\right]-c_{0}(s)\right\}>0\right\} \\
& \geqslant \mathbf{P}\left\{Y_{\alpha} \circ \Lambda(t)>c_{0}(t)\right\}=\mathbf{P}\left\{\Lambda^{1 / \alpha}(t) Y_{\alpha}(1)>c_{0}(t)\right\} \\
& =\int_{0}^{\infty} \mathbf{P}\left\{\Lambda(t)>\left(\frac{c_{0}(t)}{x}\right)^{\alpha}\right\} d \Phi_{\alpha}(x) \\
& =\int_{0}^{\infty} \bar{Q}_{t}\left(\left(\frac{c_{0}(t)}{x}\right)^{\alpha}\right) d \Phi_{\alpha}(x)=: \underline{\psi}\left(u_{0}, t\right) .
\end{aligned}
$$

Here we have used again the self-similarity of the extremal process $Y_{\alpha}$. Thus, we get finally

$$
\underline{\psi}\left(u_{0}, t\right) \leqslant \psi\left(u_{0}, t\right) \leqslant \bar{\psi}\left(u_{0}, t\right) .
$$


Remember that our initial insurance model was described by the point process $\mathscr{N}$ with the associated risk process $R(t)$. Let us denote the corresponding ruin probability by $\Psi(u, t)$ with $u=c(0)$. Then

$$
\begin{aligned}
\Psi(u, t) & =\mathbf{P}\left\{\inf _{0 \leqslant s \leqslant t}\left(c(s)-\sum_{k=1}^{N(s)} X_{k}\right)<0\right\} \\
& =\mathbf{P}\left\{\inf _{0 \leqslant s \leqslant t}\left(u_{n}^{-1} \circ c(s)-\sum_{k=1}^{N(s)} X_{n k}\right)<0\right\} \\
& =\mathbf{P}\left\{\inf _{0 \leqslant s \leqslant \tau_{n}^{-1}(t)}\left(u_{n}^{-1} \circ c \circ \tau_{n}(s)-\sum_{k=1}^{k_{n}\left(\theta_{n}(s)\right)} X_{n k}\right)<0\right\} .
\end{aligned}
$$

Now let the initial capital $u$ and time $t$ increase with $n \rightarrow \infty$ in such a way that $u /\left(k_{n}^{1 / \alpha} L\left(k_{n}\right)\right)=u_{0}, \tau_{n}^{-1}(t)=t_{0}$. Recall $c^{(n)}(t)=u_{n}^{-1} \circ c \circ \tau_{n}(t)$. Observe that under conditions a) $-f)$ we may approximate

$$
\Psi(u, t) \approx \psi\left(u_{0}, t_{0}\right)
$$

and consequently for $u$ and $t$ «large enough»

$$
\underline{\psi}\left(u_{0}, t_{0}\right) \leqslant \Psi(u, t) \leqslant \bar{\psi}\left(u_{0}, t_{0}\right) .
$$

$\mathrm{Ex} \mathrm{a} \mathrm{m} \mathrm{p} \mathrm{l} \mathrm{e} \mathrm{6.} \mathrm{Assume} \mathrm{our} \mathrm{insurance} \mathrm{model} \mathrm{is} \mathrm{characterized} \mathrm{by}$

a) the claim size distribution function $F \in N D A\left(S_{1 / 2}(1,1,0)\right)$; this means that $G_{\alpha}(x)=2(1-\Phi(\sqrt{1 / x}))$ is the Lévy distribution function; here $\Phi$ is the standard normal distribution function; hence we have to choose $u_{n}(x)=n^{2} x$;

b) the claim times process which is determined by $k(t)=[t]$ and $\theta(t) \stackrel{d}{=}$ $t \theta(1)$ with $\theta(1)$ uniformly distributed in $[0,1]$.

Then we have to choose $\tau_{n}(t)=n t$ and consequently $Q_{t}(x)=x / t$. Furthermore, since $c^{(n)}(t)=n^{-2} c(n t) \rightarrow c_{0}(t)$ we take $c_{0}(t)=u_{0}+t^{2}$. Then

$$
\begin{aligned}
& \underline{\psi}\left(u_{0}, t_{0}\right)=\int_{u_{0} / t_{0}^{2}+1}^{\infty}\left(1-\frac{1}{t_{0}} \sqrt{\frac{u_{0}+t_{0}^{2}}{x}}\right) d\left(e^{-1 / \sqrt{x}}\right), \\
& \bar{\psi}\left(u_{0}, t_{0}\right)=\int_{u_{0} / t_{0}^{2}}^{\infty}\left(1-\frac{1}{t_{0}} \sqrt{\frac{u_{0}}{x}}\right) d G_{\alpha}(x) .
\end{aligned}
$$

5. Appendix. Here we give numerical results related to the computation of (4.5) and (4.6) in the last example. Let us consider first (4.5). For the numerical computation, it is necessary to find a sufficiently large constant $K_{1}$ such that

$$
\underline{\psi}_{\varepsilon}\left(u_{0}, t_{0}\right)=\int_{u_{0} / t_{0}^{2}+1}^{K_{1}(\varepsilon)}\left(1-\frac{1}{t_{0}} \sqrt{\frac{u_{0}+t_{0}^{2}}{x}}\right) d\left(e^{-1 / \sqrt{x}}\right)
$$


and

$$
\left|\underline{\psi}\left(u_{0}, t_{0}\right)-\underline{\psi}_{\varepsilon}\left(u_{0}, t_{0}\right)\right|<\varepsilon,
$$

where $\varepsilon>0$ is arbitrarily small. We start by observing that

$$
\left|\underline{\psi}\left(u_{0}, t_{0}\right)-\underline{\psi}_{\varepsilon}\left(u_{0}, t_{0}\right)\right|<\int_{K_{1}}^{\infty} d\left(e^{-1 / \sqrt{x}}\right)=1-e^{-1 / \sqrt{K}_{1}} .
$$

Hence, if $K_{1}(\varepsilon)=(1 / \ln (1-\varepsilon))^{2}$, we achieve the desired accuracy. Moreover, since

$$
\left|\underline{\psi}\left(u_{0}, t_{0}\right)-\underline{\psi}_{\varepsilon}\left(u_{0}, t_{0}\right)\right|>\left(1-\frac{1}{t_{0}} \sqrt{\frac{u_{0}+t_{0}^{2}}{K_{1}}}\right) \int_{K_{1}}^{\infty} d\left(e^{-1 / \sqrt{x}}\right)
$$

we can find upper and lower bounds of the approximation error:

$$
\begin{aligned}
\left(1-\frac{1}{t_{0}} \sqrt{\frac{u_{0}+t_{0}^{2}}{K_{1}(\varepsilon)}}\right)\left(1-e^{-1 / \sqrt{K}_{1}(\varepsilon)}\right) & <\left|\underline{\psi}\left(u_{0}, t_{0}\right)-\underline{\psi}_{\varepsilon}\left(u_{0}, t_{0}\right)\right| \\
& <1-e^{-1 / \sqrt{K}_{1}(\varepsilon)}
\end{aligned}
$$

Concerning (4.6), we arrive at similar results using the same reasoning and the asymptotic behavior of the tail of $\alpha$-stable random variable $X$ (see, e.g., [12]):

$$
\lim _{\lambda \rightarrow \infty} \lambda^{\alpha} \mathbf{P}\{X>\lambda\}=C_{\alpha} \frac{1+\beta}{2} \sigma^{\alpha}
$$

The lower and upper bounds of the approximation error in the particular case of Lévy distribution $G_{\alpha}$ are the following:

$$
\left(1-\frac{1}{t_{0}} \sqrt{\frac{u_{0}}{K_{2}(\varepsilon)}}\right)\left(1-G_{\alpha}\left(K_{2}(\varepsilon)\right)\right)<\left|\bar{\psi}\left(u_{0}, t_{0}\right)-\bar{\psi}_{\varepsilon}\left(u_{0}, t_{0}\right)\right|<1-G_{\alpha}\left(K_{2}(\varepsilon)\right)
$$

where

$$
K_{2}(\varepsilon)=\left(\frac{C_{1 / 2}}{\varepsilon}\right)^{2}
$$

and

$$
\bar{\psi}_{\varepsilon}\left(u_{0}, t_{0}\right)=\int_{u_{0} / t_{0}^{2}}^{K_{2}(\varepsilon)}\left(1-\frac{1}{t_{0}} \sqrt{\frac{u_{0}}{x}}\right) d G_{\alpha}(x) .
$$

Acknowledgment. The Appendix is due to Stoyan Stoyanov, graduate student at Sofia University, e-mail: stoyan.stoyanov@finanalytica.com. E. Pancheva and S. Stoyanov are partially supported by the Bulgarian Ministry of Education and Sciences under grant MM 1103/2001. E. Pancheva also takes pleasure in thanking CIMAT, Guanajuato for the hospitality during her stay there. E. Kolkovska thanks Conacyt grant C 02-42522 for a 
support. We also wish to express our deep gratitude to an anonymous referee for his useful comments that led to an improvement of the paper.

Table 1. $\underline{\psi_{\epsilon}}\left(u_{0}, t_{0}\right), \epsilon=10^{-6}$

\begin{tabular}{|c|c|c|c|c|c|c|c|c|c|}
\hline \multicolumn{2}{|c|}{} & \multicolumn{10}{|c|}{$t_{0}$} \\
\cline { 3 - 10 } \multicolumn{1}{|c|}{} & 2.3 & 2.8 & 3.3 & 4.8 & 5.8 & 6.8 & 7.8 & 8.8 \\
\hline & 1 & 0.3454 & 0.3522 & 0.3564 & 0.3623 & 0.3640 & 0.3651 & 0.3657 & 0.3662 \\
& 1.61 & 0.3337 & 0.3436 & 0.3499 & 0.3590 & 0.3617 & 0.3634 & 0.3644 & 0.3652 \\
& 2.84 & 0.3137 & 0.3283 & 0.3380 & 0.3527 & 0.3573 & 0.3601 & 0.3619 & 0.3631 \\
& 4.06 & 0.2971 & 0.3151 & 0.3273 & 0.3468 & 0.3530 & 0.3568 & 0.3594 & 0.3612 \\
& 5.29 & 0.2831 & 0.3034 & 0.3177 & 0.3411 & 0.3489 & 0.3537 & 0.3570 & 0.3592 \\
& 6.52 & 0.2710 & 0.2931 & 0.3090 & 0.3358 & 0.3449 & 0.3507 & 0.3546 & 0.3573 \\
& 7.74 & 0.2604 & 0.2838 & 0.3009 & 0.3307 & 0.3411 & 0.3477 & 0.3522 & 0.3554 \\
& 8.97 & 0.2511 & 0.2754 & 0.2936 & 0.3259 & 0.3374 & 0.3449 & 0.3500 & 0.3536 \\
$u_{0}$ & 10.19 & 0.2427 & 0.2677 & 0.2867 & 0.3213 & 0.3339 & 0.3421 & 0.3477 & 0.3517 \\
& 11.42 & 0.2352 & 0.2607 & 0.2804 & 0.3169 & 0.3304 & 0.3394 & 0.3455 & 0.3500 \\
& 12.65 & 0.2284 & 0.2543 & 0.2745 & 0.3127 & 0.3271 & 0.3367 & 0.3434 & 0.3482 \\
& 13.87 & 0.2222 & 0.2483 & 0.2690 & 0.3087 & 0.3239 & 0.3341 & 0.3413 & 0.3465 \\
& 15.1 & 0.2164 & 0.2428 & 0.2638 & 0.3048 & 0.3208 & 0.3316 & 0.3393 & 0.3448 \\
& 16.32 & 0.2112 & 0.2376 & 0.2589 & 0.3011 & 0.3178 & 0.3292 & 0.3372 & 0.3431 \\
& 17.55 & 0.2063 & 0.2328 & 0.2544 & 0.2975 & 0.3148 & 0.3268 & 0.3353 & 0.3415 \\
& 18.77 & 0.2017 & 0.2283 & 0.2500 & 0.2941 & 0.3120 & 0.3244 & 0.3333 & 0.3398 \\
& 20 & 0.1975 & 0.2240 & 0.2459 & 0.2908 & 0.3092 & 0.3222 & 0.3314 & 0.3382 \\
\hline
\end{tabular}

Table 2. $\overline{\psi_{\epsilon}}\left(u_{0}, t_{0}\right), \epsilon=10^{-6}$

\begin{tabular}{|c|c|c|c|c|c|c|c|c|c|}
\hline \multicolumn{2}{|c|}{} & \multicolumn{10}{|c|}{$t_{0}$} \\
\cline { 3 - 9 } & 1 & 2.3 & 2.8 & 3.3 & 4.8 & 5.8 & 6.8 & 7.8 & 8.8 \\
\hline \multirow{6}{*}{} & 1.6563 & 0.7156 & 0.7583 & 0.8338 & 0.8624 & 0.8827 & 0.8977 & 0.9093 \\
& 1.61 & 0.5748 & 0.6425 & 0.6941 & 0.7889 & 0.8253 & 0.8510 & 0.8701 & 0.8848 \\
& 2.84 & 0.4735 & 0.5440 & 0.6023 & 0.7204 & 0.7683 & 0.8023 & 0.8277 & 0.8472 \\
& 4.06 & 0.4115 & 0.4796 & 0.5386 & 0.6673 & 0.7231 & 0.7635 & 0.7938 & 0.8172 \\
& 5.29 & 0.3687 & 0.4335 & 0.4912 & 0.6241 & 0.6851 & 0.7304 & 0.7648 & 0.7915 \\
& 6.52 & 0.3370 & 0.3985 & 0.4543 & 0.5880 & 0.6523 & 0.7014 & 0.7391 & 0.7686 \\
& 7.74 & 0.3122 & 0.3707 & 0.4246 & 0.5574 & 0.6237 & 0.6755 & 0.7159 & 0.7479 \\
& 8.97 & 0.2922 & 0.3480 & 0.4000 & 0.5310 & 0.5984 & 0.6521 & 0.6948 & 0.7288 \\
& 10.19 & 0.2756 & 0.3291 & 0.3792 & 0.5080 & 0.5759 & 0.6310 & 0.6754 & 0.7112 \\
& 11.42 & 0.2615 & 0.3129 & 0.3613 & 0.4876 & 0.5556 & 0.6117 & 0.6574 & 0.6947 \\
& 12.65 & 0.2494 & 0.2988 & 0.3458 & 0.4695 & 0.5373 & 0.5940 & 0.6408 & 0.6793 \\
& 13.87 & 0.2388 & 0.2866 & 0.3321 & 0.4533 & 0.5206 & 0.5776 & 0.6253 & 0.6649 \\
& 15.1 & 0.2295 & 0.2757 & 0.3198 & 0.4386 & 0.5054 & 0.5626 & 0.6108 & 0.6513 \\
& 16.32 & 0.2212 & 0.2659 & 0.3089 & 0.4252 & 0.4914 & 0.5486 & 0.5973 & 0.6385 \\
& 17.55 & 0.2137 & 0.2572 & 0.2990 & 0.4130 & 0.4785 & 0.5356 & 0.5846 & 0.6263 \\
& 18.77 & 0.2069 & 0.2492 & 0.2900 & 0.4017 & 0.4666 & 0.5234 & 0.5726 & 0.6148 \\
& 20 & 0.2008 & 0.2419 & 0.2817 & 0.3914 & 0.4555 & 0.5120 & 0.5613 & 0.6039 \\
\hline
\end{tabular}




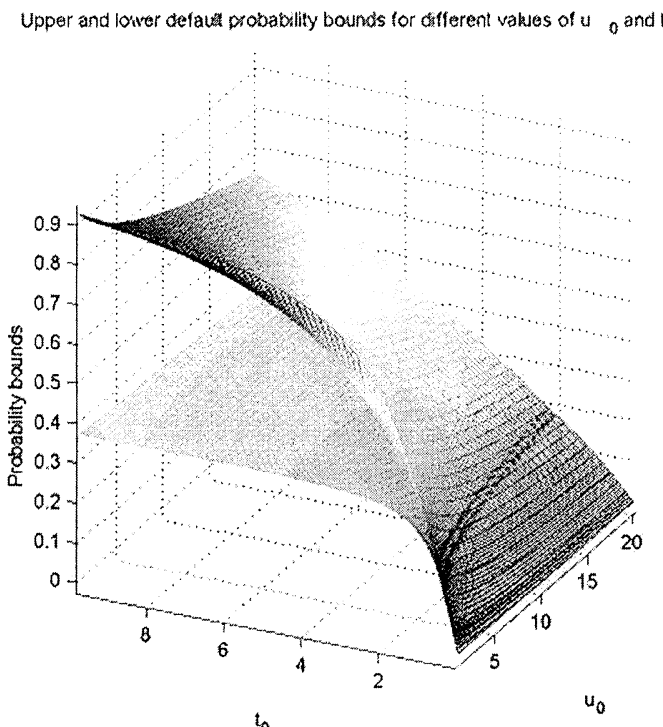

$t_{0}$

$u_{0}$

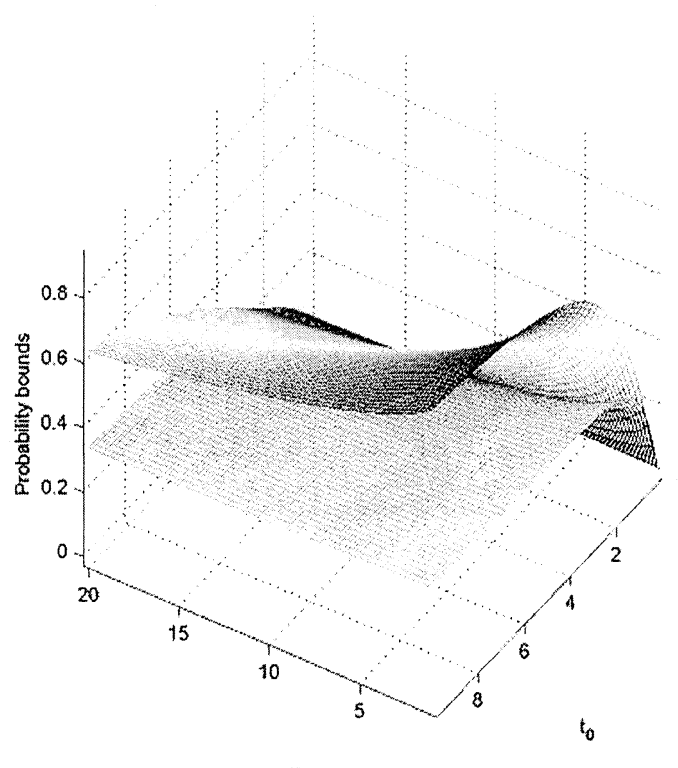

u

1. Balkema A. A., Resnick S. I. Max-infinite divisibility. - J. Appl. Probab., 1977, v. 14, № 2, p. 309-319.

2. Balkema A. A., Pancheva E. I. Decomposition for multivariate extremal processes. Comm. Statist. Theory Methods, 1996, v. 25, № 4, p. 737-758.

3. Bingham N.H. Limit theorems for occupation times of Markov processes. Z. Wahrscheinlichkeitstheor. Verw. Geb., 1971, v. 17, p. 1-22.

4. Furrer H., Michna Z., Weron A. Stable Lévy motion approximation in collective risk theory. - Insurance Math. Econ., 1997, v. 20, № 2, p. 97-114. 
5. Гнеденко Б. В., Гнеденко Д. Б. О распределениях Лапласа и логистическом как предельных в теории вероятностей. - Сердика, т. 8, № 2, с. 229-234.

6. Гнеденко Б. В., Фахим Г. Об одной теореме переноса. - Докл. АН СССР, 1969, т. 187 , № 1 , c. $15-17$.

7. Maejima M., Sato K., Watanabe T. Remarks on semi-selfsimilar processes. Research report KSTS/RR-97/013. Tokyo: Keio University, 1997.

8. Mikosch T., Resnick S. Activity rates with very heavy tails. - Stochastic Process. Appl., 2006, v. 116, № 2, p. 131-155.

9. Resnick S. Extreme Values, Regular Variation and Point Processes. New York: Springer-Verlag, 1987, $320 \mathrm{p}$.

10. Pancheva E. I. Self-similar extremal processes. - J. Math. Sci. (New York), 1998, v. 92 , № 3, p. 3911-3920.

11. Pancheva E. I. Semi-selfsimilar extremal processes. - J. Math. Sci. (New York), 2000, v. 99 , № 3, p. 1306-1316.

12. Samorodnitsky G., Taqqu M.S. Stable non-Gaussian random processes. New York: Chapman \& Hall, 1994, 632 p.

13. Satheesh $S$. Aspects of randomization in infinitely divisible and max-infinitely divisible laws. - Probab. Statist. Models, 2002, v. 1, p. 7-16.

14. Silvestrov D. S., Teugels J. L. Limit theorems for extremes with random sample size. Adv. in Appl. Probab., 1998, v. 30, № 3, p. 777-806.

15. Whitt W. Some useful functions for functional limit theorems. - Math. Oper. Res., 1980 , v. 5, № 1, p. 67-85.

Поступила в редакцию

11.VIII. 2003

Исправленный вариант

15.IV.2005 